\title{
Collective effects in cellular structure formation mediated by compliant environments: a Monte Carlo study
}

\author{
Ilka B. Bischofs* and Ulrich S. Schwarz \\ University of Heidelberg, Im Neuenheimer Feld 293, \\ D-69120 Heidelberg, Germany

\begin{abstract}
Max Planck Institute of Colloids and Interfaces, D-14424 Potsdam, Germany
\end{abstract}

\begin{abstract}
Keywords: cell-matrix adhesion, mechanotransduction, tissue organization, elastic interactions
\end{abstract}

${ }^{*}$ Present address: Department of Bioengineering, Center for Synthetic Biology, University of California, Berkeley, CA 94720-3224, USA 


\begin{abstract}
Compliant environments can mediate interactions between mechanically active cells like fibroblasts. Starting with a phenomenological model for the behaviour of single cells, we use extensive Monte Carlo simulations to predict non-trivial structure formation for cell communities on soft elastic substrates as a function of elastic moduli, cell density, noise and cell position geometry. In general, we find a disordered structure as well as ordered string-like and ring-like structures. The transition between ordered and disordered structures is controlled both by cell density and noise level, while the transition between string- and ring-like ordered structures is controlled by the Poisson ratio. Similar effects are observed in three dimensions. Our results suggest that in regard to elastic effects, healthy connective tissue usually is in a macroscopically disordered state, but can be switched to a macroscopically ordered state by appropriate parameter variations, in a way that is reminiscent of wound contraction or diseased states like contracture.
\end{abstract}




\section{Introduction}

In order to develop and maintain tissues, cells in multicellular organisms have to interact with each other and the extracellular matrix (ECM). Cellular communication proceeds mainly through specific interactions provided by receptor-ligand binding. In solution, gradients in ligand concentration encode spatial information. For example, morphogen gradients guide cell differentiation [1], and gradients of chemoattractants or chemorepellants direct cell motility (chemotaxis) [2]. For cells adhering to each other or to the ECM, physical properties of the environment like topography and mechanics provide additional information supplementing specific biochemical cues. In particular, cells in their natural environment typically orient along fiber bundles of the ECM, a principle termed contact guidance [3]. In general, cells preferentially orient along directions with minimal curvature [4]. While contact guidance provides only a bidirectional cue for cell migration, unidirectional cues result from spatial gradients in adhesiveness (haptotaxis) [5], substrate rigidity (durotaxis) [6] and substrate tension (tensotaxis) [7, 8].

Tissue cells like fibroblasts in the connective tissue constantly remodel their structural environment by degrading old and secreting new ECM. Moreover they can exert forces large enough to actively reorganize the ECM after it has been laid down [9]. Hence, cells not only respond to the physical properties of their environment, but also actively modulate them. This results in indirect, matrix-mediated interactions between cells. In particular, cell traction-induced reorganization of collagen fibers can mediate a mechanical interaction between cells via contact guidance [10, 11]. In the same vein,

elastic interactions between cells can result from stress and strain in the 
ECM induced by cell traction. During recent years, the sophisticated use of elastic substrates has proven that cells indeed respond to purely elastic features in their environment, including rigidity, rigidity gradients and prestrain [12, 6, 13, 14, 15, 16. It now appears that many cell types, including fibroblasts, smooth muscle cells, and endothelial cells (but not neutrophils or neurons), respond to the mechanical properties of their environment with a common preference for large effective stiffness [17, 18, 19]. Here the term effective stiffness comprises both rigidity of and tensile strain in the environment, which can be actively sensed by cells via mechanotransductory processes at cell-matrix adhesions [20, 21, 22, 23]. These mechanical cues play an important role in a variety of physiological processes, including development, tissue maintenance, angiogenesis, myotube fusion, wound healing and metastasis [24, 25, 26, 27]. In particular, if coupled to cell division, stress and strain become major determinants of tissue morphogenesis [28, 29]. In general, tissue function arises from the close relationship between cell behaviour in and material properties of the ECM [30, 31, 32].

We have argued before that elastic interactions contribute to the way single cells position and orient themselves in compliant environments [33, 34, 35]. However, it has not been discussed in detail before how this individual behaviour translates into collective behaviour of cell ensembles in compliant environments. In a recent short report we have shown that elastically interacting cells assemble into strings of cells which then leads to screening of the cellular traction patterns [36]. Although this effect in principle suppresses macroscopic order, we have also found that macroscopic order can exist at high cell density, with an interesting competition between string- and ring- 
like structures as a function of the Poisson ratio of the surrounding material. In our recent report, this effect has been especially analyzed for ordered (lattice) arrangements of cells, which can be decomposed into strings. Since cellular systems are intrinsically noisy, in this paper we focus on stochastic effects using extensive Monte Carlo simulations, which allow to predict collective effects in the presence of noise. In the following, we mainly consider the case of cells adhering to the top of a soft elastic substrate, because this setup might be the easiest way to experimentally verify our theoretical predictions. Commonly used materials for elastic substrates are polyacrylamide or polydimethylsiloxane, which can be described by linear isotropic elasticity theory. Since we are interested in the interactions mediated by an elastic environment, we only consider situations without cell-cell contact, which experimentally could trigger different cellular responses to mechanical signals. Therefore in our theoretical work we first fix cell positions and then relax cellular orientations using standard Monte Carlo techniques. Experimentally, this might be done by using microcontact printing, thus restricting cells to adhesive islands, or by using non-motile cell lines, which adhere at random positions and then do not move. By freezing in cell positions, we are also able to control cell density. For the position geometry, we consider ordered (lattice) arrangements, random perturbations around ordered positions and arrangements which are completely random. We calculate structural phase diagrams as a function of of elastic moduli, cell density, noise and cell position geometry using Monte Carlo simulations. We then briefly investigate collective elastic effects in three dimensional elastic environments and finally discuss our results in the context of wound healing. 


\section{Model and simulations}

\subsection{General concepts}

In order to sense the mechanical properties of their environment, tissue cells pull on it with actomyosin contractility. In many cases, this contractile mechanical activity is directed along the long axis of the cell body, especially when confronted with a mechanically anisotropic environment. The mechanical action of such an anisotropic force pattern can be modeled as an anisotropic force contraction dipole $P_{i j}=P l_{i} l_{j}$, where the unit vector $\vec{l}$ specifies cell orientation. In contrast to electric dipoles in electrostatics, which are vectors, force dipoles in elasticity theory are tensors of rank two. $P$ specifies the dipole strength, which typically has $|P|=F d \approx 10^{-11} \mathrm{~J}$, corresponding to two opposing forces $F=200 \mathrm{nN}$ separated by a distance $d=60 \mu \mathrm{m}$ [37. We will consider cells with anisotropic force patterns only and assume that the magnitude $P$ is constant for all cells. A mechanically active cell generates an elastic strain field $u_{i j}(\vec{r})$, which again is a tensor of rank two. The strain induced by $P$ can be calculated from the elastic Green tensor $G_{i j}\left(\vec{r}, \vec{r}^{\prime}\right)$ which describes the deformation of the medium at $\vec{r}$ caused by a point force at $\vec{r}^{\prime}$. In general $G_{i j}$ depends on material properties and boundary conditions. For translationally invariant situations, one has $G_{i j}\left(\vec{r}, \vec{r}^{\prime}\right)=G_{i j}\left(\vec{r}-\vec{r}^{\prime}\right)$. Elastic interactions between two cells result if the mechanical activity $P_{i j}$ of one cell responds to the elastic field $u_{i j}$ induced by another cell. In the framework of a general field theory elastic interaction can then be represented by a coupling of $u_{i j}$ and $P_{i j}$. Since cells are active and moreover often show a regulated response, the exact form of the coupling 
between $P_{i j}$ and $u_{i j}$ cannot be predicted from first principles. In a first order approximation we may assume that $P_{i j}$ and $u_{i j}$ are linearly coupled. For mechanically active cells like fibroblasts, experimental observations suggest that indeed they adopt positions and orientations in such a way as to effectively minimize the scalar quantity $W=P_{i j} u_{i j}$ [34, 35]. For tensile strain, this implies that contractile cells actively align with the external field. Because they pull against the external stretch, cells reduce displacement. For fibroblastlike cells, this behaviour might have evolved in the context of wound healing, when cell traction is required to close wounds. A biophysical interpretation of the origin of the extremum principle regarding $W$ is to note that $W$ has dimensions of energy. Physically $W$ can be interpreted as an extra energy contribution to the deformation energy required to build up a force dipole in the presence of strain $u_{i j}$ [34, 35]. Using the analogy of a harmonic spring the basic idea can be explained easily: for a given spring constant $K$, it takes the work $W=F^{2} /(2 K)$ to build up the force $F$. Therefore larger stiffness $K$ corresponds to smaller work $W$ required to reach the force $F$. In this way $W$ may be interpreted as the inverse of an effective stiffness of the environment and the extremum principle in $W$ corresponds to the experimental observation that cell-matrix contacts grow stronger in a stiff environment, thus eventually determining cell orientation. Using this extremum principle, we have been able before to unify many diverse observations which have been reported for the organization of single cells on elastic substrates and in physiological hydrogels [34, 35]. For example, it predicts that single cells prefer to align in parallel and perpendicular to free and clamped surfaces of finite sized samples, respectively. It is however important to note that the 
cellular response to the actively sensed effective stiffness of the environment is very different from the cellular response to cyclic external stretch with a $1 \mathrm{~Hz}$ frequency, which is relevant for the physiology of lung tissue and the cardiovascular system. In this case, many cell types tend to orient away from the direction of stretch [38, 39], possibly to avoid the recurrent deformation of their cytoskeleton.

For two cells, the above reasoning leads to the following effective interaction potential [34, 35]

$$
W=P_{i j} u_{i j}=-\sum_{i, j, k, l} P_{i j} \frac{\partial}{\partial x_{j}} \frac{\partial}{\partial x_{l}} G_{i k}\left(\vec{r}-\vec{r}^{\prime}\right) P_{k l}^{\prime}
$$

where we consider only situations with translational invariance. The optimal cell configuration is described by the minimum of $W$ in regard to cell positioning and orientation. Since in general $G_{i k}$ scales as $\sim 1 /(E r)$, where $r$ is distance and $E$ an elastic modulus, $W$ scales as $\sim P^{2} /\left(E r^{3}\right)$ and has units of energy. Since in a linear material the cellular strain fields superimpose, the functional that describes elastic interactions of a system of $N$ cells reads:

$$
W=\frac{1}{2 N} \sum_{\gamma=1}^{N} \sum_{\delta \neq \gamma}^{N} W_{\gamma \delta},
$$

where $W_{\gamma \delta}$ is the interaction between two cells $\gamma$ and $\delta$ as described in Eq. 10 The factor $1 / 2$ is required to avoid double counting and the factor $1 / N$ for per cell normalization. The optimal structure is given by the configuration which minimizes Eq. 2 as a function of all cellular orientations and positions and we may refer to this structure as the ground state, in analogy to interacting passive particles in physical systems. 


\subsection{Monte Carlo simulations}

In the presence of noise any system will deviate from its optimal state. In the cellular systems discussed here, noise results both from intracellular processes (like the intrinsic stochasticity of gene expression and signal transduction) and from heterogeneities in the material properties of the environment. In order to introduce a stochastic element into the structure formation process, we perform Monte Carlo simulations using the standard Metropolis algorithm to generate typical configurations in the presence of noise [40]. This implies the use of an Gibbs ensemble, which in our case is the simplest choice given that we do not know the exact details of the cellular decision making process (in information theory, the Gibbs ensemble arises from maximizing the measure for disorder (Shannon entropy) under the constraint of a prescribed average energy). Starting from an arbitrary configuration, a cell is selected at random and its orientation randomly varied. The new configuration is always accepted when it decreases $W$. Otherwise it is accepted with the probability $\exp \left(-\Delta W / k_{B} T\right)$, where $k_{B}$ is the Boltzmann constant and $T$ is temperature. In our context $k_{B} T$ represents a measure for the degree of stochasticity involved in cellular decision making. Other studies on modelling cellular structure formation have used the same concept before and found an effective value of $k_{B} T=510^{-15} \mathrm{~J}$ [41, 42, 43]. Since this quantity comprises all molecular interactions between a cell and its environment, it is six orders of magnitudes larger than thermal energy. In our case, the competition between elastic and stochastic effects can be characterized by the reduced temperature

$$
T^{\star}=\frac{k_{B} T \pi E b^{3}}{P^{2}}
$$


which measures the relative importance of noise with respect to the average elastic interaction strength. Here $b$ is the average distance between two cells which is related to the averaged cell density by $\langle\rho\rangle=1 / b^{2}$ in two dimensions (2D) and by $\langle\rho\rangle=1 / b^{3}$ in three dimensions (3D). As we will see below, typical values in our simulations range from $T^{\star}=0.1$ to 3.0. Using $P=10^{-11} \mathrm{~J}, E=10 \mathrm{kPa}, b=100 \mu \mathrm{m}$ and a typical value $T^{\star}=1$, we obtain $k_{B} T=310^{-15} \mathrm{~J}$. Therefore our choice for the noise level is exactly in the same range as the earlier estimates [41, 42, 43]. As the effective temperature is decreased towards cero, $T^{\star} \rightarrow 0, W$ decreases and the ground states become increasingly favorable. This process is known as simulated annealing and is often used to identify optimal structures [4]. In the vicinity of an optimal structure, almost all Monte Carlo moves are rejected. In contrast, when $T^{\star} \rightarrow \infty$, every Monte Carlo move is accepted and the structures get disordered. One Monte Carlo sweep corresponds to $N$ such Monte Carlo moves. After thermal equilibration (typically about $10^{3}-10^{4}$ Monte Carlo sweeps), the Metropolis algorithm samples the important configurations typical for a given temperature $T^{\star}$. One can then calculate statistical properties of structures at defined noise level by averaging the quantity of interest over many configurations generated by the Metropolis algorithm.

To study stochastic effects using our Monte Carlo simulations we typically consider $N \approx 1000$ dipoles. In order to minimize the effects of boundaries and finite size, we apply periodic boundary conditions (pbcs), such that each dipole has the same number of next neighbors and experiences the same local geometry. We implement pbcs using the minimal image convention, i.e. we only consider the interactions of the dipole with its $N-1$ nearest 
(image) dipoles [40]. Note that in the two-dimensional situation of cells on top of an elastic half space, the $1 / r^{3}$ interaction effectively constitutes a short-ranged potential, because its area integral does not diverge with system size $\left(\int^{L} r d r\left(1 / r^{3}\right) \sim 1 / L\right.$ where $L$ is system size). Thus, boundary effects are expected to play only a minor role in this case and the minimal image convention is a good approximation.

\subsection{Additional assumptions}

We now make some additional assumptions which will simplify our subsequent calculations and which will allow for a direct comparison of our theoretical calculations with appropriate experiments. First, we assume that the environment can be described by isotropic linear elasticity theory, which is a reasonable assumption for the synthetic substrates like the ones made from polyacrylamide or polydimethylsiloxane commonly used to study mechanical effects in cell culture [45, 37]. Thus there are two elastic constants: the Young modulus $E$ describes the rigidity of the material and the Poisson ratio $\nu$ the relative importance of compression and shear. In particular we mainly consider the situation of cells adhering to the top surface of a thick elastic film. Such a situation can be theoretically represented by an elastic half-space geometry with dipolar orientations constrained to the $\mathrm{x}-\mathrm{y}$-plane. Therefore the Young modulus $E$ and the Poisson ratio $\nu$ are $3 \mathrm{D}$ quantities. The maximal value for $\nu$ is $1 / 2$, e.g. for strongly hydrated polymer gels. If such a material is tensed in one direction, the shear mode is excited and it contracts in the perpendicular directions (Poisson effect). For common materials, the minimal value for the Poisson ratio is $\nu=0$, e.g. in dehydrated 
fibrous polymer gels. Then the volume mode prevails and uniaxial tension does not translate into lateral contraction. For cells exerting tangential forces on top of an elastic substrate one can use the Boussinesq Green function of an elastic halfspace [4] to specify Eq. 11 to [35]:

$$
W_{\gamma \delta}=\frac{a_{1} P^{2}}{r^{3}} f\left(\theta, \theta^{\prime}, \alpha\right)
$$

where $r$ is the distance between cells and the cellular orientations $\theta, \theta^{\prime}$ and $\alpha$ are defined via the scalar products $\cos \theta=\vec{l} \cdot \vec{r}, \cos \theta^{\prime}=\overrightarrow{l^{\prime}} \cdot \vec{r}$ and $\cos \alpha=\vec{l} \cdot \overrightarrow{l^{\prime}}$. $f$ is given by

$$
\begin{aligned}
f\left(\theta, \theta^{\prime}, \alpha\right) & =3\left(\cos ^{2} \theta+\cos ^{2} \theta^{\prime}-5 \cos ^{2} \theta \cos ^{2} \theta^{\prime}-\frac{1}{3}\right) \\
& -\left(1-a_{2}\right) \cos ^{2} \alpha-3\left(a_{2}-3\right) \cos \alpha \cos \theta \cos \theta^{\prime}
\end{aligned}
$$

where $a_{1}=\nu(1+\nu) /(\pi E)$ and $a_{2}=(1-\nu) / \nu$.

Secondly, because here we focus on elastic effects, we want to avoid cellcell contacts, which are known to change the mechanical state of adhering cells [16. Therefore we attribute an exclusion disc of radius $a$ to each cell. Moreover in most of our simulations cell positions are frozen and only orientational degrees of freedom are considered. Experimentally, this situation might be achieved by using non-motile cell lines or appropriate drugs to suppress cell motility. Alternatively, one might use microcontact printing to prepare well-defined adhesive islands constraining cell positions.

Finally, while the focus of our paper is on structure formation on planar synthetic substrates to allow a direct comparison of theory and experiment, we also briefly consider cells in a three-dimensional (3D) environment. For computational simplicity we keep the assumption of linear isotropic. For cells 
embedded in an infinite 3D isotropic elastic environment, the interaction law stays the same as Eq. 5 , but with different constants $a_{1}=(1+\nu) /(8 \pi E(1-\nu))$ and $a_{2}=(3-4 \nu)$ which now follow from the Kelvin solution for the full elastic space [46]. In 3D, the elastic interactions are truly long-ranged, in the sense that now the volume integral over the elastic interaction diverges with system size $\left(\int^{L} r^{2} d r\left(1 / r^{3}\right) \sim \ln L\right.$ where $L$ is system size), thus boundary effects become more important.

\section{Results and Discussion}

\subsection{Structure formation for dipolar lattices}

\subsubsection{Phase diagrams under thermal noise}

We first consider structure formation in the presence of noise when cells adopt regular positions on an infinite square (s) and hexagonal (h) lattice. In general we find a strong dependence of structure formation on lattice geometry, Poisson ratio $\nu$ and noise level $T^{\star}$. In particular our simulations show an interesting competition between string-like and ring-like structures at low noise levels, which we have also found before in a detailed analysis of optimal structures [36. For the square lattice low noise structures are always string-like. The lattice geometry favors the formation of strings directed along one of the principal lattice vectors $\vec{n}=(1,0)$ or $\vec{n}=(0,1)$ for all $\nu$. The limit $\nu \rightarrow 0$ shows structural degeneracy, that is, additional structures, e.g. parallel strings aligned along $\vec{n}=(1,1)$, become equally favored as parallel (1,0)-string structure. Enhanced structural degeneracy of string-like 
structures in the limit of vanishing Poisson ratio occurs also on the hexagonal lattice. Most interestingly, however, on the hexagonal lattice stringand ring-like structures exchange stability as a function of Poisson ratio. For $\nu>0.32$ the Poisson effect favors ring-like structures over strings, with rings composed of four dipoles each rotated by 90 degrees relatively to its neighbor. On both lattice types the orientational order imposed by elastic interactions is gradually destroyed with increasing noise levels. When elastic interactions dominate, dipoles typically fluctuate weakly around their optimal orientations forming long-ranged ordered structures. At intermediate noise levels, long-ranged order is decreased, but there are still ordered domains which locally adjust to elastic signals. As noise increased further, domain size decreases and local fluctuations become stronger, eventually giving eventually rise to completely random dipole arrangements.

The loss of long-ranged order with increased noise can be quantified by calculating the temperature behavior of appropriate order parameters. For string-like structures all dipoles point along a common direction $\vec{n}$. A suitable parameter to quantify the degree of global alignment therefore seems to be the angle $\beta$ between dipole orientation $\vec{l}$ and $\vec{n}$, where $\cos \beta=\vec{l} \cdot \vec{n}$. Note that force dipoles have a bipolar symmetry and thus dipole orientations $\vec{l}$ and $-\vec{l}$ are equivalent. Thus, the average $\langle\cos \beta\rangle=0$. However, the average $\left\langle\cos ^{2} \beta\right\rangle$ becomes one when all dipoles point along $\vec{n}$, while for an isotropic structure $\left\langle\cos ^{2} \beta\right\rangle=\frac{1}{2}$. Thus, we define an alignment order parameter $p$ as

$$
p=2\left\langle\cos ^{2} \beta\right\rangle-1
$$

which is non-zero only for globally aligned structures and zero otherwise. From our Monte Carlo simulations we compute $p$ as a function of $T^{\star}$ and 
Poisson ratio for both lattice types. As shown in Fig. 1ha, on the square lattice a globally aligned structure $p \rightarrow 1$ always develops regardless of Poisson ratio when the noise level becomes sufficiently low. The transition temperature $T_{c}^{\star}$ from a state without into a state with long-ranged orientation correlations, i.e. where $p \neq 0$ for the first time, decreases approximately linearly with $\nu$. This might be attributed to the general enhancement of elastic interactions with increasing Poisson ratio $W \sim(1+\nu)$. In Fig. 10 we show how the probability distribution to find a dipole with a given orientation $\beta$ evolves with increasing noise levels. At low $T^{\star}$ almost all dipoles point along $\vec{n}$ and the orientation distribution is strongly peaked; with increasing $T^{\star}$ the distribution broadens and finally for $T^{\star}>T_{c}^{\star}$ all orientations are - on a global scale - equally likely (circle). In contrast to the square lattice, where long-ranged order always implies alignment, the type of long-ranged order developed on a hexagonal lattice depends on Poisson ratio. Fig. 11 shows that an aligned structure develops only for $\nu<\nu_{c} \approx 0.32$. Interestingly, in this case the relation between the transition temperature $T_{c}^{\star}$ and Poisson ratio is also reversed compared to the square lattice: $T_{c}^{\star}$ stays roughly constant for $\nu<0.2$ and then drops quickly around $\nu \approx \nu_{c}$. Moreover, towards $\nu_{c}$ the transition seems to become more discontinuous and in our simulations $p$ suddenly jumps from a low to a large value, indicating bistability and a first order phase transition. The $T_{c}^{\star}$ scaling with $\nu$ might be explained by simultaneous overall decrease in elastic signal strength with decreasing $\nu$ within strings compensated by an increase in lateral string-string coupling favoring global alignment at small $\nu$. For larger Poisson ratio $(\nu \geq 0.4)$ we find $p=0$ at all temperatures. At low $T^{\star}<T_{c}^{\star}$ a macroscopic isotropic 
long-ranged ring-like order exists build up by small rings composed of four dipoles oriented at 90 degrees with respect to each other. That is, at $T^{\star}<T_{c}$ there are two equally large subpopulations of dipoles oriented at 90 degrees with respect to each other. This corresponds to the two peaks in the radial orientation distribution shown in Fig. 11d $(\nu=0.5)$. For $\nu=0.5$ the transition temperature into the ordered ring-like structure $T_{c}^{\star}$ is slightly larger than 1 and significantly lower than $T_{c}^{\star} \approx 2$ observed for the string structures on the square lattice at equal dipole densities.

In 2 and 3 we summarize our results for ordered structures in the form of $\nu-T^{\star}$ phase diagrams for square and hexagonal lattices, respectively. We have included representative snapshots of typical dipole configurations at noise levels well below $T_{c}^{\star}$ in the globally ordered state, approximately at the phase transition $T_{c}^{\star}$ and well above $T_{c}^{\star}$ in the disordered phase for $\nu=0.1$ and $\nu=0.5$, respectively. It is important to note that despite the loss of global order at elevated noise levels $T^{\star}>T_{c}^{\star}$, elastic interactions still locally manifest themselves in characteristic domain formation. For example, at $T^{\star}=3$ elastic interactions still cause local order on a square lattice and one finds short string-like domains preferentially oriented along the principal lattice vectors. On incompressible substrates $(\nu=0.5)$ we furthermore often observe cooperative excitations and the formation of ring-like domains. This may reflect the competition between ring- and string-like domains which is modulated by the Poisson ratio. In fact, as we have shown before, at $\nu=0.5$ ring-like structures are only slightly disfavoured with respect to the string-like structures [36]. Nevertheless, it may seem surprising that ring-like domains are excited so easily already at very low $T^{\star}$ even before coexistence of the 
equally favorable $(1,0)$ and $(0,1)$ domains occurs (see snapshot at $T^{\star}=1$ ). The reason is probably a large domain interface penalty for two $(1,0)$ and $(0,1)$ string domains compared to the interface penalty of a string and an adjacent ring-domain.

For the hexagonal lattice local structural characteristics of elastic interactions under noise are again string-like domains preferentially oriented along directions specified by lattice geometry. For large $\nu$, a characteristic signature for elastic interactions is the formation of 4-cell-ring or ladder-domains with cells having approximately perpendicular orientations with respect to each other. Thus, although experimentally noise might be too strong to allow for global ordering, elastic interactions might be still detected by their local signatures of characteristic domain formation.

\subsubsection{Effect of weak positional disorder}

Experimentally cells may not adopt perfect lattice positions, in particular when adhesive islands are used which are large compared to cell size in order to minimize the effects of island shape on cellular force distribution [47, 48]. We therefore investigate the effect of positional fluctuations at low thermal noise intensities. For this purpose we randomly displace the dipole positions within a circle of radius $r$ around the lattice positions before relaxing the orientations. The degree of positional disorder can be quantified by the ratio $f=r / b$ of the radius with respect to the lattice constant $b$. In the following we focus on the square lattice and $\nu=0.5$, since synthetic polymer gels typically have $\nu \approx 0.5$. For this value of the Poisson ratio, Fig. 1 predicts a lattice geometry dependent transition from a string-like structure on a square 
lattice to a ring-like structure on the hexagonal lattice. However, rings are thermally excited easily on the square lattice. Hence the string-like structure might also be easily destabilized under positional disorder.

In Fig. 4 we show Monte Carlo snapshots at a low reduced temperature $\left(T^{\star}=0.1\right)$ for $f=0.1,0.25,0.5$. The simulations show that a $10 \%$ deviation from the lattice positions has only a minor effect on orientational ordering. For $f=0.25$, string-like as well as ring-like domains form and long-ranged orientational order disappears. As for thermal noise, ring-domains typically localize to interfaces between string-like domains with perpendicular orientations. With increasing $f$ domains shrink and structures appear increasingly disordered. Thus, moderate deviations from the square lattice positions are not sufficient to destabilize string-like structures with respect to ring-like structures on incompressible substrates. Possibly because under uniform positional noise dipoles still find themselves in a local fourfold coordination with other dipoles (that is, each dipole has typically for next neighbors as on the square lattice), string domains are stabilized. We conclude that positional disorder affects structure formation in a similar way as increasing the temperature $T^{\star}$ on a perfect lattice.

\subsection{Phase behavior on homogeneous substrates}

On a homogeneous substrate, non-motile cells usually adhere more or less at random positions. Therefore we next study typical structures on elastic substrates with completely randomized but fixed positions as described above. Cellular structure formation on elastic substrates is now governed mainly by three control parameters, namely reduced temperature $T^{\star}$, Poisson ratio $\nu$ 
and cell density. Since in our model each cell is characterized by an exclusion radius $a$, we introduce a reduced density

$$
\rho^{\star}=\frac{N \pi a^{2}}{L^{2}},
$$

which is a dimensionless variable describing the ratio of the area occupied by $N$ circular disks of radius $a$ to the area of the (simulation) box with length $L$.

Fig. 5 shows typical snapshots of structures at $T^{\star}=0.1$ for cells on an elastic substrate with $\nu=0,0.25,0.35,0.5$ (top-bottom) for $\rho^{\star}=0,0.4,0.5$ (left-right). At low densities cells predominantly optimize locally the interaction between them by forming short strings with no long-range correlation between string-like clusters. We attribute this finding to the strong tendency of dipoles to form strings and the strong elastic screening of stringstring interactions [36]. This results in rather robust pattern formation that does not differ qualitatively as the Poisson ratio is varied, see Fig. 5 . One expects that these patterns represent typical cellular structures formed by strongly interacting cells when cells in dilute concentrations are suspended on an elastic substrate and adhere at random positions $\left(\rho^{\star} \rightarrow 0\right)$. With increasing $\rho^{\star}$ the respective structures at low noise intensity show a strong dependence on the Poisson ratio $\nu$. For incompressible substrates $(\nu=0.5)$ we find isotropic ring-like structures, see Fig. $5(\mathrm{IVc})$. With decreasing Poisson ratio string-like patterns emerge. For $\nu=0.35$ we find coexistence of string-like and ring-like domains, compare Fig. 或(IIIc). For substrates with Poisson ratio below $\nu<0.32$ string-like structures dominate at intermediate densities. With increasing density strings start to interact and domains of aligned parallel strings form which increase in size with increasing $\rho^{\star}$, see 
Fig. 15(IIb,IIc). Highly compressible substrates $(\nu \rightarrow 0)$ favor cell alignment along a common direction at high cell densities and in Fig. 5(Ic) we find a globally aligned structure. Here, the rotational symmetry of the structure is spontaneously broken along an arbitrary direction in space despite lacking long-ranged positional order. In this respect we may speak of a nematic structure in analogy to nematic phases formed by liquid crystals which are characterized by orientational order but positional disorder.

As before one can quantify the development of anisosotropic long-ranged order with the order parameter $p$ defined in Eq. 6. In contrast to the lattice structures where preferred string direction $\vec{n}$ was determined by lattice geometry, the orientation of the director $\vec{n}$ on homogeneous substrates is arbitrary. This is taken into account by defining a two-dimensional analog of the nematic order parameter $p$ used in the theory of liquid crystals [49. We first introduce the ordering matrix $Q_{i j}=\frac{1}{N} \sum_{\alpha=1}^{N}\left(l_{i}^{\alpha} l_{j}^{\alpha}-\frac{1}{2} \delta_{i j}\right)$, where $\vec{l}^{\alpha}$ is the orientation vector of the $\alpha^{6}$ th particle and the sum runs over all particles in the simulation box. The largest eigenvalue $\lambda$ of the symmetric ordering matrix $Q$ corresponds then to the order parameter $p=2 \lambda$. As before $p$ measures the degree of orientational order with respect to the current director $\vec{n}$, which is the corresponding eigenvector to the maximal eigenvalue. $p=1$ implies nematic and $p=0$ isotropic structures. Since we freeze in positions, we first thermally average $p$ for a fixed configuration of the dipole positions and subsequently average over at least 20 random position configurations obtained for the same $\rho^{\star}$. In Fig. 6] we show the quantitative effects of the reduced density $\rho^{\star}$, Poisson ratio $\nu$ and reduced temperature $T^{\star}$ on nematic ordering. At constant temperature $T^{\star}=0.1$ a nematic structure 
forms beyond a critical density $\rho_{c}^{\star}(\nu)$ and below a critical value of the Poisson ratio, see Fig. 6a. The degree of structural alignment increases with increasing $\rho^{\star}$ and approaches $p \rightarrow 1$ toward the maximal $\rho^{\star}=0.907$, which is the maximal packing density achieved for disks arranged on a hexagonal lattice. For $\nu \geq 0.4$ no nematic ordering $p=0$ occurs at any density $\rho^{\star}$ or temperature $T^{\star}$, because ordered structures are ring-like. In addition to the density-dependent ordering transition, the nematic structure is also destabilized by increased noise. In Fig. 6b we plot $p$ for $\nu=0$ at different values of the reduced temperature $T^{\star}$. The critical density $\rho_{c}^{\star}$ to obtain a nematic structure increases with $T^{\star}$ and $p=0$ for any any $\rho^{\star}$ when $T^{\star}$ exceeds a critical $T_{c}^{\star} \approx 1$, which corresponds approximately to $T_{c}^{\star}$ measured on the hexagonal lattice.

In Fig. 6re we show the phase diagram in the $\nu$ - $\rho^{\star}$-plane for $T^{\star}=0.1$. Diamonds mark $p<0.4$, squares $p>0.4$ and the dashed line denotes our estimate for the isoline $p=0.4$. We find three different phases: a high-density anisotropic string-like structure, a high-density isotropic ring-like structure and a low density disordered structure. The general features of this phase diagram are very similar to the thermal phase diagram for hexagonal lattices shown in Fig. 3 but with the role of $T^{\star}$ and (inverse) density $\rho^{\star}$ exchanged. Note, that at $\rho^{\star}=0.907$ dipoles in fact adopt hexagonal lattice positions, because disks must order into a hexagonal lattice due to the non-overlap constraint. Thus, reducing the density from $\rho^{\star}=0.907$ in our model is in some sense equivalent to introducing position fluctuations around hexagonal lattice positions (see above), which qualitatively has similar consequences as raising the effective temperature. These results also show the importance 
of local position geometry and dipole coordination. At high $\rho^{\star}$ dipoles have six next neighbors and all interactions are equal in magnitude. Because elastic interactions are strongly anisotropic and dependent on Poisson ratio, non-trivial structure formation as a function of local dipole coordination and Poisson ratio results. Decreasing $\rho^{\star}$ the number of next neighbors decreases and at low $\rho^{\star}$ dipoles typically only have one or two next neighbors, which favors dipole alignment and string formation. Due to screening of the traction patterns in a string, adjacent strings hardly interact and we obtain a largely disordered structure even at low $T^{\star}$. In our calculations the degree of position correlation is controlled by $\rho^{\star}$, which therefore plays a similar role as lattice geometry before. In contrast to relative density effects described by $\rho^{\star}$, absolute density effects can be subsumed into the effective temperature $T^{\star}$, see Eq. 3. This is due to the fact that by increasing cell density the screening efficiency of string-string interactions also increases. The only way to overcome screening and introduce lateral coupling between dipoles is to insure that dipole-dipole distances within a string and the distance to an adjacent string are comparable. Increased dipole coordination, however, is controlled by $\rho^{\star}$ rather than by $\rho$.

We note that the order-disorder transition with $\rho^{\star}$ might also represent a spin-glass transition. Such transitions are common for magnets, where spins represent magnetic moments. In magnets global ordering of spins can result in a ferromagnetic state, where all spins point along a common direction and a macroscopic magnetic field builds up. Anti-ferromagnetic order results when spins are globally ordered but with equal numbers of up and down spins, such that no macroscopic polarization builds up. A spin-glass 
transition occurs when e.g. due to disordered positions of spins no ferroor anti-ferromagnetic order develops even at low temperatures. In analogy, dipoles in our model represent spins. The aligned structure therefore might be called a ferroelastic phase, because a macroscopically anisotropic polarization stress develops spontaneously. In analogy, the ring-like structure might be called an antiferroelastic phase, because the macroscopic stress exerted by the dipoles remains isotropic. When $\rho^{\star}<\rho_{c}$, no global order develops even in the limit $T^{\star} \rightarrow 0$; instead, many equally favorable states exist. Because we do not allow dipoles to adjust positions, they cannot relax into an ordered ferro- or anti-ferroelastic state. Thus, the disordered low temperature phase might also represent a spin glass. There is an interesting difference between thermally disordered states and density disordered states at low T. In both cases, dipoles can be aligned by application of an external field. The former might be called a paraelastic phase because it relaxes into the unpolarized disordered state after the external field is switched off with a single time-scale $\tau \propto\left(T-T_{c}\right)^{\gamma}$. In contrast, the later might represent a spin-glass state and relaxation into the disordered state cannot be described by a single power law. In fact a characteristic feature of spin glasses is a fast relaxation to a remanent polarization and then a slow relaxation into the unpolarized state, which may exceed experimental timescales.

\section{Elastic effect in 3D environments}

Although cell behaviour in $3 \mathrm{D}$ is not the focus of this paper, we now spell out a few predictions resulting from our model in this case. Calculations 
for cells positioned on a simple cubic lattice suggest that in incompressible environments $(\nu=1 / 2)$ the optimal structure is effectively isotropic with a hedgehog-like unit cell, where all dipoles at the corners point to the cube's center, see Fig. 17, while for $\nu=0$ spontaneous symmetry breaking along a principal lattice lattice vector occurs, see Fig. [7b. This indicates that similar transitions between ring-like and string-like structures as a function of Poisson ratio $\nu$ exist in $3 \mathrm{D}$ as they do in 2D. String-like structures might also be favored by anisotropies in the effective mechanical properties of the environment. For example, fiber alignment or external strain fields might cause cell alignment with the external perturbation, which could be further stabilized by elastic interactions between cells. In Fig. 7f we show a corresponding snapshot of a Monte Carlo simulation with 100 cells modeled as hard spheres with an elastic dipole moment at their center. Here we allowed for both orientational and positional degrees of freedom $\left(T^{\star}=2, \nu=1 / 2\right)$, where the position dependence of $W$ from Eq. 2 is used for additional Monte Carlo moves. Moreover we applied a homogeneous strain field along the $z$-direction. Cells respond by an alignment with the external field and the formation of strings as has been observed experimentally [50]. Without external field but with mobile cells we typically observe formation of "networks" of cells at low temperature and intermediate densities and disconnected uncorrelated strings at elevated noise levels and low densities, respectively. In general, we expect that the low to intermediate density regime of a three 3D phase diagram for mobile elastically interacting force dipoles will resemble the phase behavior of electric dipolar fluids [53, 54]. In both systems string formation dominates and string-string interactions are screened in the same 
way [55]. At high dipole densities our lattice calculations in 3D suggest that phase transitions as a function of Poisson ratio may occur and we find evidence that again a low Poisson ratio of the material tends to favor global cell alignment.

In finite $3 \mathrm{D}$ environments the geometry and boundary condition of the sample may also affect structure formation. The presence of a boundary modifies the direct elastic interaction between cells by boundary induced strain fields, which, depending on boundary condition, introduce either attractive or repulsive contributions to the elastic interaction plus a direct interaction term with the boundary [34, 35]. An instructive example for the competition between the direct interaction of a cell with the boundary and cellular interactions is the elastic half space with a clamped boundary. When cells are located close to the boundary, the direct interaction with a clamped boundary favors cellular orientations pointing toward the surface [34, 35]. On the other hand, interactions between cells favor the formation of strings and thus parallel orientations. Our calculations suggest that the transition between these two configurations is a function of the ratio $\alpha=b / d$, where $d$ is the distance to the boundary and $b$ is the distance between cells, and the number of interacting cells $N$. Above a critical value $\alpha_{c} \approx 2.3$ the direct interaction with the boundary dominates and cells are expected to point toward the surface. For $\alpha<\alpha_{c}$ orientations in parallel to the clamped surface are favored when sufficiently many cells are present, $N>N_{c}(\alpha)$. In Fig. [7] we plot $N_{c}$ as a function of $\alpha$. Thus, collective effects may alter preferred cell organization close to clamped boundaries. In contrast for a free surface the direct interaction with boundary favors parallel alignment, which is further 
stabilized by collective elastic effects between cells. This may explain the robust parallel alignment of cells with respect to free surfaces which has been observed experimentally [51, 52].

\section{Conclusion and Outlook}

Using a simple mathematical model and extensive Monte Carlo simulations, we have analyzed structure formation due to elastic effects for fibroblast-like cells with anisotropic force patterns. Our model predicts in a quantitative way how structure formation is controlled on soft elastic substrates by the Poisson ratio $\nu$, the reduced cell density $\rho^{\star}$, the relative strength of elastic interactions with respect to noise (represented by the reduced temperature $\left.T^{\star}\right)$ and the geometry of cell positioning. Up to intermediate cell densities, we predict the formation of uncorrelated strings of cells (paraelastic phase). This finding can be explained by noting that the cellular traction patterns in strings screen each other, as we have demonstrated before by an analytical calculation [36]. For high cell densities, we find an interesting competition between isotropic ring-like and anisotropic string-like structures. The string-like (ferroelastic) phase is reminiscent of the nematic phase for liquid crystals. In contrast, the nematic order parameter vanishes in the ring-like (anti-ferroelastic) phase, because local ordering principles preclude nematic order on the macroscopic scale.

The Poisson ratio $\nu$ allows to switch between these phases. It strongly affects cellular structure formation due to the non-trivial way in which stress and strain propagates in the elastic environment. The antiferroelastic phase 
is expected to occur on synthetic elastic substrates made from e.g. polyacrylamide or polydimethylsiloxane, which are characterized by $\nu \approx 1 / 2$. In order to obtain the nematic phase, new kinds of polymer gels for cell culture are needed, with considerable lower values for the Poisson ratio (namely below $\nu_{c}=0.32$ ). One possible route might be the design of biocompatible polymer gels with large meshsizes but little hydration, thus avoiding the incompressibility effect of bound water. As it is an open issue on which time and length scales cells sense the mechanical properties of their environment [19], design and testing of new materials is crucial for progress in this field.

Like the Poisson ratio, also the other determinants of cellular structure formation are in principle accessible experimentally. The Young modulus $E$ directly affects the reduced temperature and can easily be varied in experiments. Nevertheless it does not appear to be a reasonable control variable for experiments, mainly because the predicted structure formation might be expected to take place only in a small range of $E$-values, namely the ones corresponding to physiological rigidities (around $\mathrm{kPa}$ ). Other control parameters of interest are cellular contractility (which affects $T^{\star}$ through the force dipole moment $P$ and which might be varied for example by administering LPA, which stimulates Rho-mediated contractility) and the average distance $b$ between cells (although, in order for the force dipole approximation to remain valid, the typical distance between cells should not be smaller than the typical cell length). The most important experimental control parameter next to the Poisson ratio $\nu$ might be the geometry of cell positioning, which can be controlled with patterning techniques (including microcontact printing in two dimensions). 
Elastic effects are likely to contribute to large scale tissue organization and to the structure-function relationship of tissues. Our results indicate that for sparsely populated tissues, elastic interactions, despite their longranged character, might have only a rather local effect. This is a result of the local tendency for cells to align into strings combined with the resulting screening of elastic fields. In this regime the system remains macroscopically disordered over a wide range of variations in material properties and cell density, effectively making the composite material of matrix and cells robust against perturbations. It is well known that the sparsely populated connective tissue is a rather disordered structure. Our simulations suggest that without macroscopic external fields elastic interactions between cells favor isotropic disordered structures. In this case the average stress in the tissue resulting from cells is isotropic, that is $\sigma_{i j}=P \rho \delta_{i j}$, where $\rho$ is the cell density. The situation changes when an external field is applied (e.g. resulting from a wound). Then highly aligned structures may prevail. In this case cells orient their mechanical activity in such a way that the average cellular stress in the medium $\sigma_{i j}=\rho\left\langle P_{i j}\right\rangle$ becomes anisotropic in response to homogeneous uniaxial stress, that is, the cellular stress is directed opposite to the externally applied stress (e.g. as to close the wound). It is an interesting question what happens after the external alignment field has decreased to zero. Our calculations suggest that elastic interactions may stabilize an aligned structure even in the absence of external fields. In particular, disordered tissues (like the connective tissue) may become aligned due to an external field. Due to elastic interactions the transition from the aligned state back into the normal disordered state upon switching off the external field could be dramatically 
slowed down, in analogy to similar effects observed in magnetic spin glasses. This could have implications for wound healing and scar formation. Polarization also occurs spontaneously in our model. Interestingly, this state reminds of the disease state called contracture, when certain tissues (like skin or muscle) start to tighten up, eventually preventing movement of body parts. It is an interesting speculation whether the mechanical properties of extracellular matrices are altered by (diseased) cells in such a way as to effectively induce variations in the Poisson ratio, which could trigger transitions between ferroand anti-ferroelastic states. In order to investigate this point in more detail, our model should be extended to include viscoelastic and plastic effects as well as fiber degrees of freedom.

Mechanotaxis also plays an important role for dynamic rearrangement of groups of cells in tissues, including development, wound healing, capillary sprouting and tumor growth. The work presented here builds on experimental observations which suggest that cells migrate toward high strain areas and orient their mechanical activity in such a way as to pull back in response to external tensile strain. Such tensile strain is certainly present e.g. close to wounded areas or tumors. Future studies will show in which sense our model can be adapted to these more specific situations. The current study shows in a general way that many interesting structure formation processes arise from mechanical effects in communities of mechanosensitive cells and therefore might help not only to understand the corresponding physiological situations, but also to program new tissue functions by guiding the design of novel material with appropriate mechanical properties. 


\section{Acknowledgments}

We thank Phil Allen, Sam Safran, Assi Zemel and Thomas Pfeifer for helpful

discussions. This work was supported by the German Research Foundation (DFG) through the Emmy Noether Program as well as by the Center for Modelling and Simulation in the Biosciences (BIOMS) at Heidelberg. 


\section{References}

[1] Turing AM. The Chemical Basis of Morphogenesis. Roy Soc Lond Phil Trans Ser B 1952; 237:37-72.

[2] Berg HC, Purcell EM. Physics of chemoreception. Biophys J 1977;20:193-219.

[3] Weiss P. In vitro experiments on the factors determining the course of the outgrowing nerve fiber. J Exp Zool 1934;68:393-448.

[4] Dunn GA, Heath JP. New hypothesis of contact guidance in tissue-cells. Exp Cell Res 1976;101:1-14.

[5] Carter SB. Haptotaxis and mechanism of cell motility. Nature $1967 ; 213: 256-260$.

[6] Lo CM, Wang HB, Dembo M, Wang YL. Cell movement is guided by the rigidity of the substrate. Biophys J 2000;79:144-152.

[7] Beloussov LV, Louchinskaia NN, Stein AA. Tension-dependent collective cell movements in the early gastrula ectoderm of xenopus laevis embryos. Dev Genes Evol 2000;210:92-104.

[8] Mandeville JTH, Lawson MA, Maxfield FR. Dynamic imaging of neutrophil migration in three dimensions: Mechanical interactions between cells and matrix. J Leuk Biol 1997;61:188-200.

[9] Harris AK, Wild P, Stopak D. Silicone-rubber substrata - new wrinkle in the study of cell locomotion. Science 1980;208:177-179. 
[10] Murray JD, Oster GF, Harris AK. A mechanical model for mesenchymal morphogenesis. J Mat Biol 1983;17:125-129.

[11] Murray JD, Oster GF. Cell traction models for generating pattern and form in morphogenesis. J Mat Biol 1984;19:265-279.

[12] Pelham RJ, Wang YL. Cell locomotion and focal adhesions are regulated by substrate flexibility. Proc Natl Acad Sci USA 1997;94:13661-13665.

[13] Wong JY, Velasco A, Rajagopalan P, Pham Q. Directed movement of vascular smooth muscle cells on gradient-compliant hydrogels. Langmuir 2003;19:1908-1913.

[14] Engler AJ, Bacakova L, Newman C, Hategan A, Griffin MA, Discher DE. Substrate compliance versus ligand density in cell on gel responses. Biophys J 2004;86:617-628.

[15] Engler AJ, Griffin MA, Sen S, Bonnemann CG, Sweeney HL, Discher DE. Myotubes differentiate optimally on substrates with tissue-like stiffness: pathological implications for soft or stiff microenvironments. J Cell Biol 2004;166:877-887, 2004.

[16] Yeung T, Georges PC, Flanagan LA, Marg B, Ortiz M, Funaki M, Zahir N, Ming W, Weaver V, Janmey PA. Effects of substrate stiffness on cell morphology, cytoskeletal structure, and adhesion. Cell Motil Cytoskel 2005;60:24-34.

[17] Wong JY, Leach JB, Brown XQ. Balance of chemistry, topography and mechanics at the cell-biomaterial interface: Issues and challenges for 
assessing the role of substrate mechanics on cell response. Surface Sci 2004;570:119-133.

[18] Georges PC, Janmey PA. Cell type-specific response to growth on soft materials. J Appl Physiol 2005;98:1547-1553.

[19] Discher DE, Janmey PA, Wang YL. Tissue cells feel and respond to the stiffness of their substrate. Science 2005;310:1139-1143.

[20] Balaban NQ, Schwarz US, Riveline D, Goichberg P, Tzur G, Sabanay I, Mahalu D, Safran SA, Bershadsky A, Addadi L, Geiger B. Force and focal adhesion assembly: a close relationship studied using elastic micropatterned substrates. Nat Cell Biol 2001;3:466-472.

[21] Riveline D, Zamir E, Balaban NQ, Schwarz US, Ishizaki T, Narumiya S, Kam Z, Geiger B, Bershadsky AD. Focal contacts as mechanosensors: Externally applied local mechanical force induces growth of focal contacts by an mdia1-dependent and rock-independent mechanism. J Cell Biol 2001;153:1175-1185.

[22] Geiger B, Bershadsky A. Exploring the neighborhood: Adhesioncoupled cell mechanosensors. Cell 2002;110:139-142, 2002.

[23] Bershadsky A, Balaban NQ, Geiger B. Adhesion-dependent cell mechanosensitivity. Annu Rev Cell Dev Biol 2003;19:677-95.

[24] Galbraith CG, Sheetz MP. Forces on adhesive contacts affect cell function. Curr Opin Cell Biol 1998;10:566-571. 
[25] Huang S, Ingber DE. The structural and mechanical complexity of cellgrowth control. Nat Cell Biol 1999;1:E131-E138.

[26] Geiger B, Bershadsky A, Pankov P, Yamada KM. Transmembrane extracellular matrix-cytoskeleton crosstalk. Nat Rev Mol Cell Biol 2001;2:793-805.

[27] Bao G and Suresh S. Cell and molecular mechanics of biological materials. Nat Mat 2003;2:715-725.

[28] Shraiman BI. Mechanical feedback as a possible regulator of tissue growth. Proc Natl Acad Sci USA 2005;102:3318-23.

[29] Nelson CM, Jean RP, Tan JL, Liu WF, Sniadecki NJ, Spector AA, Chen CS. Emergent patterns of growth controlled by multicellular form and mechanics. Proc Natl Acad Sci USA 2005;102:11594-11599.

[30] Curtis A, Riehle M. Tissue engineering: the biophysical background. Phys Med Biol 2001;46:R47-R65.

[31] Griffith LG. Emerging design principles in biomaterials and scaffolds for tissue engineering. Annals NY Acad Sci 2002;961:83-95.

[32] Langer R, Tirrell DA. Designing materials for biology and medicine. Nature 2004;428:487-492, 2004.

[33] Schwarz US, Safran SA. Elastic interactions of cells. Phys Rev Lett 2002;88:048102.

[34] Bischofs IB, Schwarz US. Cell organization in soft media due to active mechanosensing. Proc Natl Acad Sci USA 2003;100:9274-9279. 
[35] Bischofs IB, Safran SA, Schwarz US. Elastic interactions of active cells with soft materials. Phys Rev E 2004;69:021911.

[36] Bischofs IB, Schwarz US. Effect of Poisson ratio on cellular structure formation. Phys Rev Lett 2005;95:068102.

[37] Schwarz US, Balaban NQ, Riveline D, Bershadsky A, Geiger B, Safran SA. Calculation of forces at focal adhesions from elastic substrate data: The effect of localized force and the need for regularization. Biophys J 2002;83:1380-1394.

[38] Shirinsky VP, Antonov AS, Birukov KG, Sobolevsky AV, Romanov YA, Kabaeva NV, Antonova GA, Smirnov VN. Mechano-chemical control of human endothelium orientation and size. J Cell Biol 1989;109:331-339.

[39] Wang JHC, Grood ES. The strain magnitude and contact guidance determine orientation response of fibroblasts to cyclic substrate strains. Conn Tissue Res 2000;41:29-36.

[40] Allen MP and Tildesley DJ. Computer simulations of Liquids. Oxford University Press, Oxford, 2001.

[41] Drasdo D, Kree R, McCaskill JS. Monte Carlo approach to tissue-cell populations. Phys Rev E 1995;52:6635-6657.

[42] Drasdo D, Forgacs G. Modeling the interplay of generic and genetic mechanisms in cleavage, blastulation, and gastrulation. Devel Dyn 2000;219:182-191. 
[43] Beysens DA, Forgacs G, Glazier JA. Cell sorting is analogous to phase ordering in fluids. Proc Natl Acad Sci USA 2000;97:9467-9471, 2000.

[44] Kirckpatrick S, Gelatt CD, Vecchi MP. Optimization by simulated annealing. Science 1983;220:671-680.

[45] Dembo M, Wang YL. Stresses at the cell-to-substrate interface during locomotion of fibroblasts. Biophys J 1999;76:2307-2316.

[46] Landau LD and Lifshitz EM. Theory of elasticity. Pergamon Press, Oxford, 1970.

[47] Parker KK, Brock AL, Brangwynne C, Mannix RJ, Wang N, Ostuni E, Geisse NA, Adams JC, Whitesides GM, Ingber DE. Directional control of lamellipodia extension by constraining cell shape and orienting cell tractional forces. FASEB J 2002;16:1195-1204.

[48] Brock A, Chang E, Ho CC, LeDuc P, Jiang XY, Whitesides GM, Ingber DE. Geometric determinants of directional cell motility revealed using microcontact printing. Langmuir 2003;19:1611-1617.

[49] de Gennes PE, Prost P. The Physics of Liquid Crystals. Oxford University Press, Oxford, 1995.

[50] Eastwood M, Mudera VC, McGrouther DA, Brown RA. Effect of precise mechanical loading on fibroblast populated collagen lattices: Morphological changes. Cell Motil Cytoskel 1998;40:13-21. 
[51] Bell E, Ivarsson B, Merrill C. Production of a tissue-like structure by contraction of collagen lattices by human-fibroblasts of different proliferative potential invitro. Proc Natl Acad Sci USA 1979;76:1274-1278.

[52] Takakuda K, Miyairi H. Tensile behaviour of fibroblasts cultured in collagen gel. Biomaterials 1996;17:1393-1397.

[53] Tlusty T, Safran SA. Defect-induced phase separation in dipolar fluids. Science 2000;290:1328-1331.

[54] Weis JJ, Levesque D. Chain formation in low density dipolar hard spheres: A Monte Carlo study. Phys Rev Lett 1993;71:2729-2732.

[55] Allen PB. Dipole interactions and electrical polarity in nanosystems: The Clausius-Mossotti and related models. J Chem Phys 2004;120:29512962. 


\section{Figure Captions}

Figure 1: Effect of noise on global alignment order. (a) The order parameter $p$ for alignment on a square lattice as a function of noise $T^{\star}$ for Poisson ratio $\nu=0,0.1,0.2,0.3,0.4,0.5$ (from left to right). For low noise, aligned structures develop for all values of the Poisson ratio. (b) The radial distribution of the dipolar orientations on a square lattice with $T^{\star}=1.5,2,3$ for Poisson ratio $\nu=1 / 2$. The two peaks correspond to an aligned structure with bipolar symmetry below $T_{C}^{\star}=3$. (c) On a hexagonal lattice, $p=0$ for $\nu=0.4,0.5$ and $p \rightarrow 1$ for $\nu=0.3,0.2,0.1,0$ from left to right. At low noise, aligned structures are unstable for large values of the Poisson ratio. (d) The radial distribution function on a hexagonal lattice with $T^{\star}=0.5,1,2$ for Poisson ratio $\nu=1 / 2$. The four peaks correspond to a ring-like structure below $T_{c}^{\star}=2$.

Figure 2: Schematic phase diagram as a function of noise level $T^{*}$ and Poisson ratio $\nu$ for the square lattice. For illustration, typical Monte Carlo snapshots are added. Both decreasing $T^{*}$ or increasing $\nu$ favors the ordered string-like phase. For $\nu=0.1$, the dipoles typically weakly fluctuate around the stringlike ground state. For $\nu=0.5$, the dominant fluctuations around the optimal state are ring-like.

Figure 3: Schematic phase diagram as a function of noise level $T^{*}$ and Poisson ratio $\nu$ for the hexagonal lattice. For illustration, typical Monte Carlo snapshots are added. Ordered phases are favored by decreasing $T^{*}$, but the ordered phases are string- and ring-like for small and large values of $\nu$, re- 
spectively.

Figure 4: Snapshots of Monte Carlo simulations at reduced temperature $T^{\star}=0.1$ for cellular force dipoles randomly displaced from an ideal square lattice for $f=0.1,0.25,0.5$ from left to right for $\nu=0.5$ (a-c) and $\nu=0$ (d-f), respectively. Increased positional disorder $f$ destroys long-ranged orientational order in a similar way as an increase in $T^{\star}$ on a perfect lattice.

Figure 5: Snapshots of Monte Carlo simulations at reduced temperature $T^{\star}=0.1$ for $N=1024$ cellular force dipoles at random, but fixed positions on an elastic substrate with $\nu=0,0.25,0.35,0.5$, respectively (top-bottom). The reduced area density increases from left to right as $\rho^{\star}=0,0.4,0.6$, respectively, while the average cell density $\langle\rho\rangle$ remains constant.

Figure 6: Averaged nematic order parameter $p$ as a function of model parameters. (a) Simulation results for $\nu=0,0.25,0.3$ (left-right) and $\nu=0.4,0.5$ (bottom), respectively. Thus nematic ordering occurs only on compressible substrates and above a critical density. (b) Simulation results for $\nu=0$ at $T^{\star}=0.1,0.6,1.1$ (top-bottom). Thus nematic ordering disappears above a certain noise level. (c) Phase diagram for $T^{\star}=0.1$ for dipoles on elastic substrates obtained by Monte Carlo simulations. All points below diamonds have $p<0.4$ and all points above squares yield $p>0.4$. The long dashed line is our estimate for the isoline with $p=0.4$. The horizontal dashed line marks the maximal $\rho^{\star}$ possible for a hexagonal lattice. 
Figure 7: Collective effects in 3D elastic media. (a,b) The optimal structure in a cubic lattice depends on the Poisson ratio $\nu$. In incompressible media $(\nu=1 / 2)$ the isotropic hedgehog structure is most favorable (a) while in highly compressible media $(\nu=0)$ elastic interactions favor aligned structures (b). (c) Cells in external strain fields form strings running along the direction of stretch due to interactions with external strain and elastic cellcell interactions. (d) Collective effects can modify preferred cell organization close to a clamped boundary, which for single cells is perpendicular. For small intercellular distances $(\alpha=b / d<2.3)$ and sufficiently many cells in a string $\left(N>N_{c}\right)$, the parallel orientation becomes more favorable. 

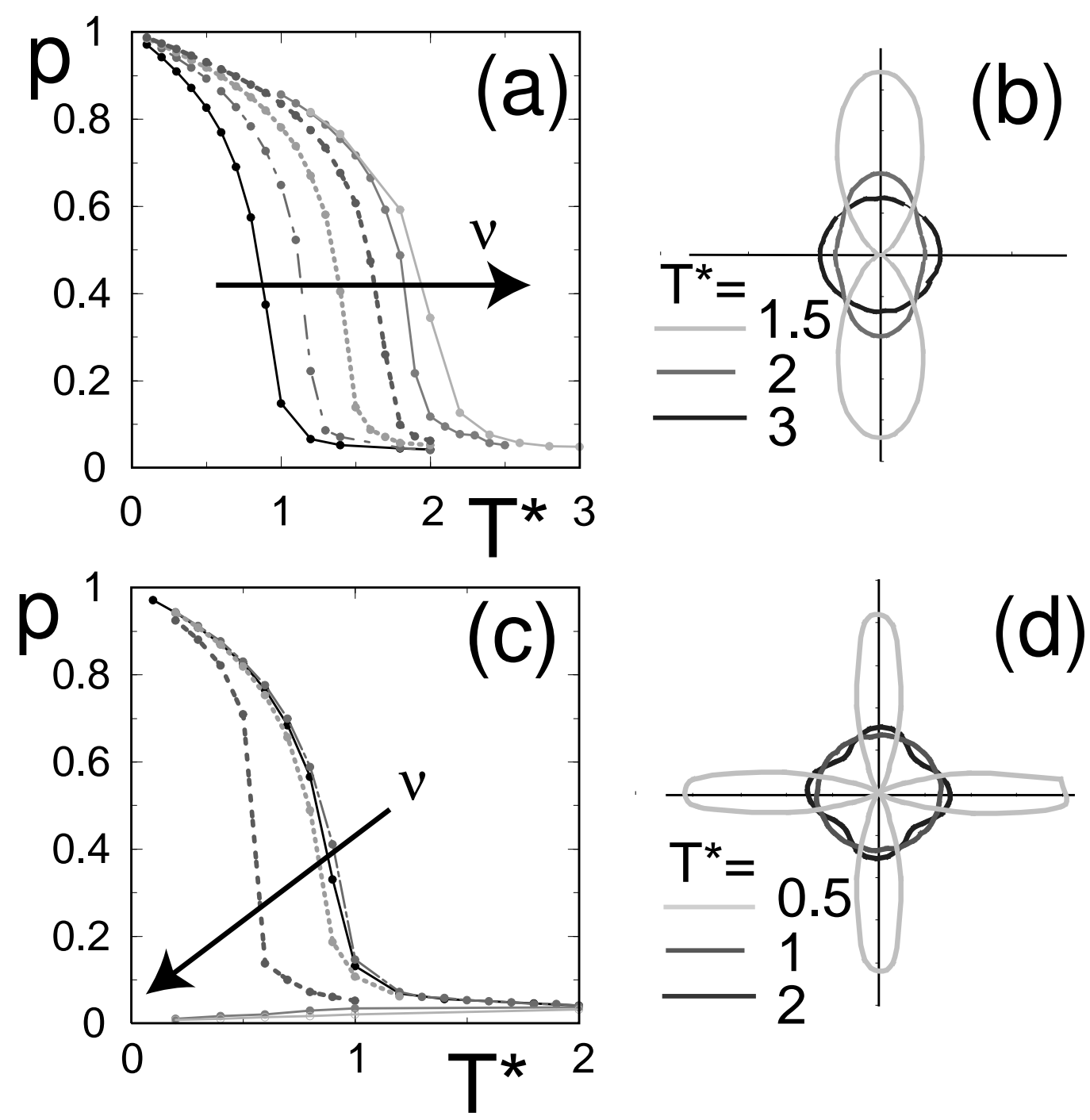

Figure 1: Bischofs and Schwarz 


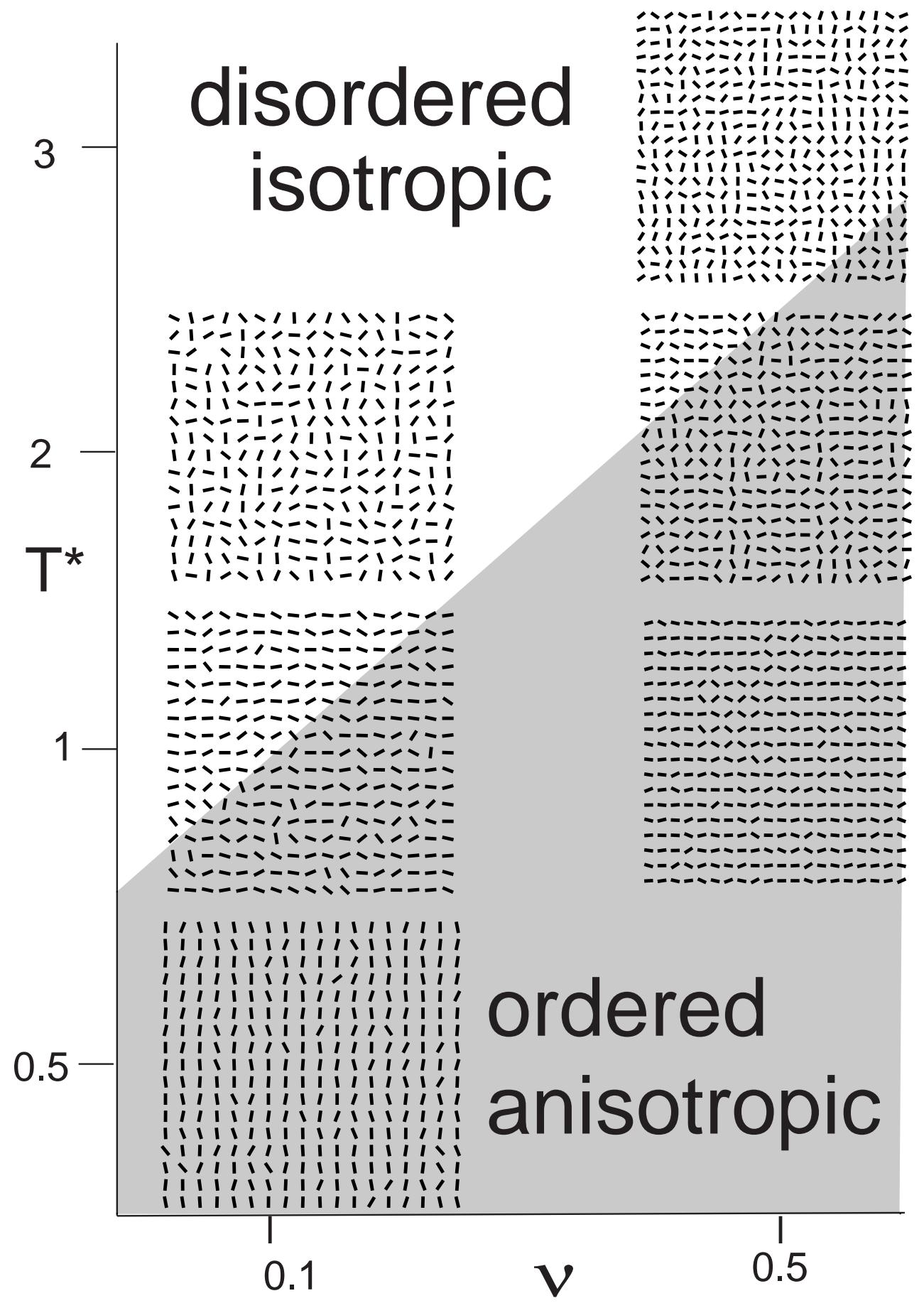

Figure 2: Bischygfs and Schwarz 


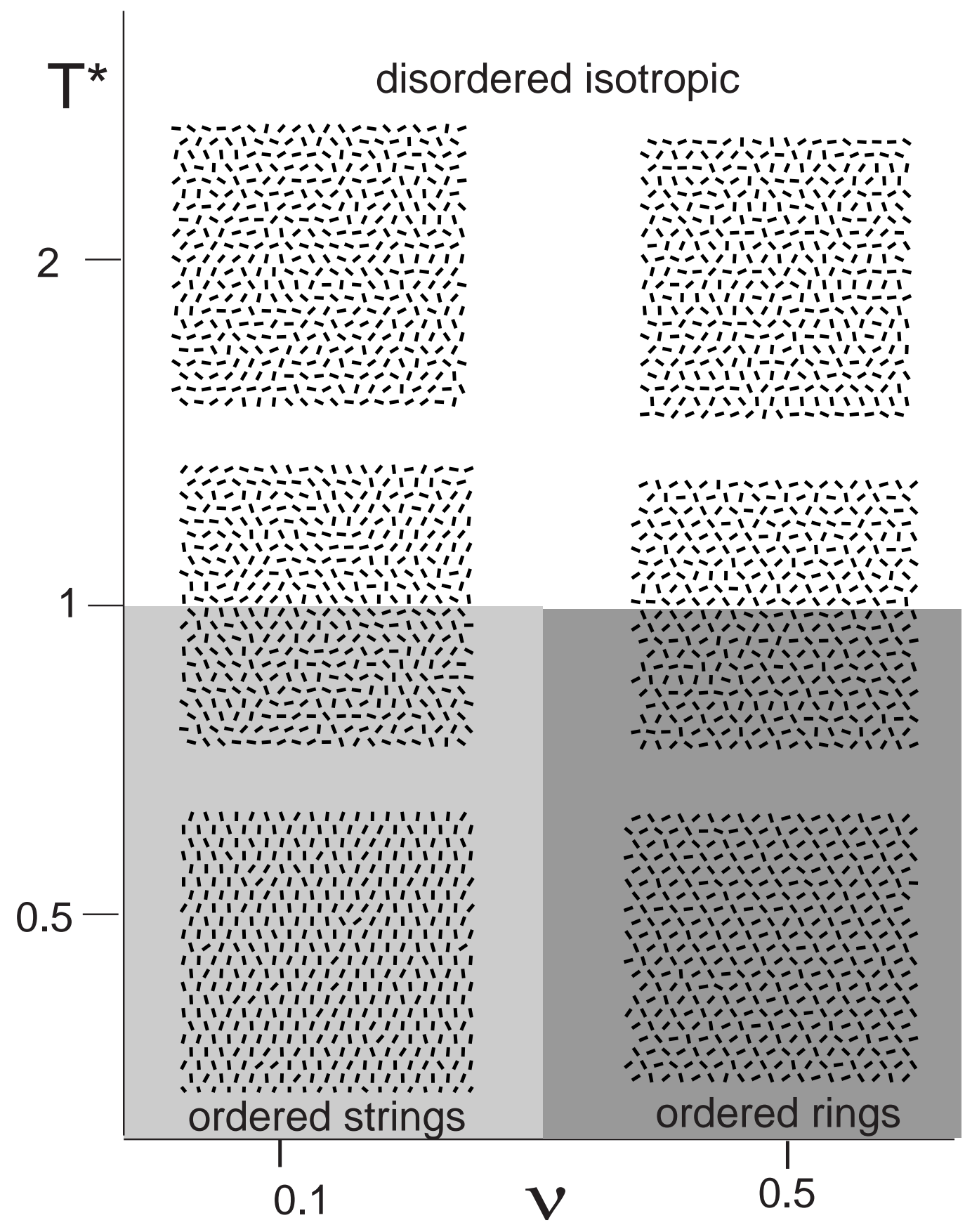

Figure 3: Bischofs and Schwarz 


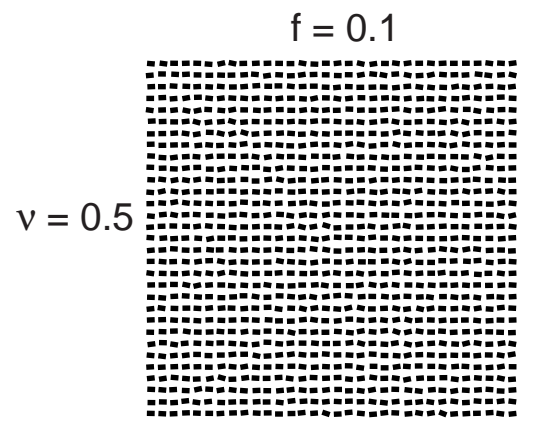

(a)

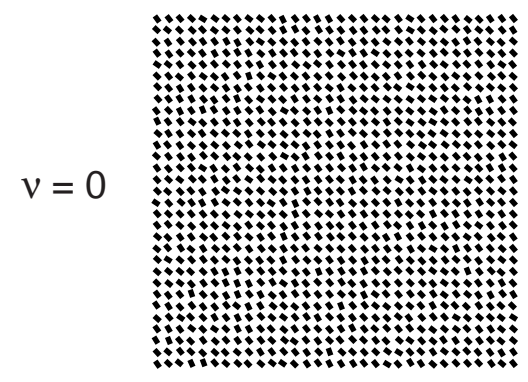

(d)

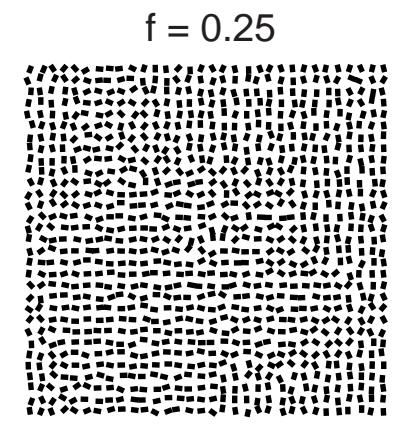

(b)

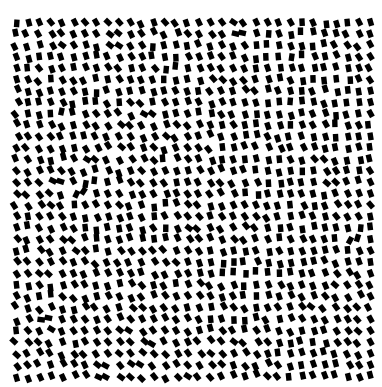

(e)

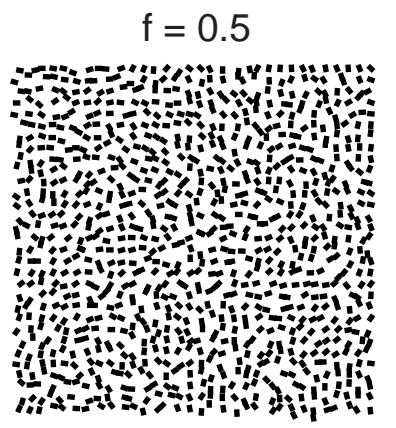

(c)

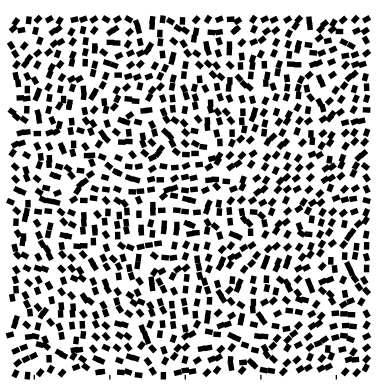

(f)

Figure 4: Bischofs and Schwarz 


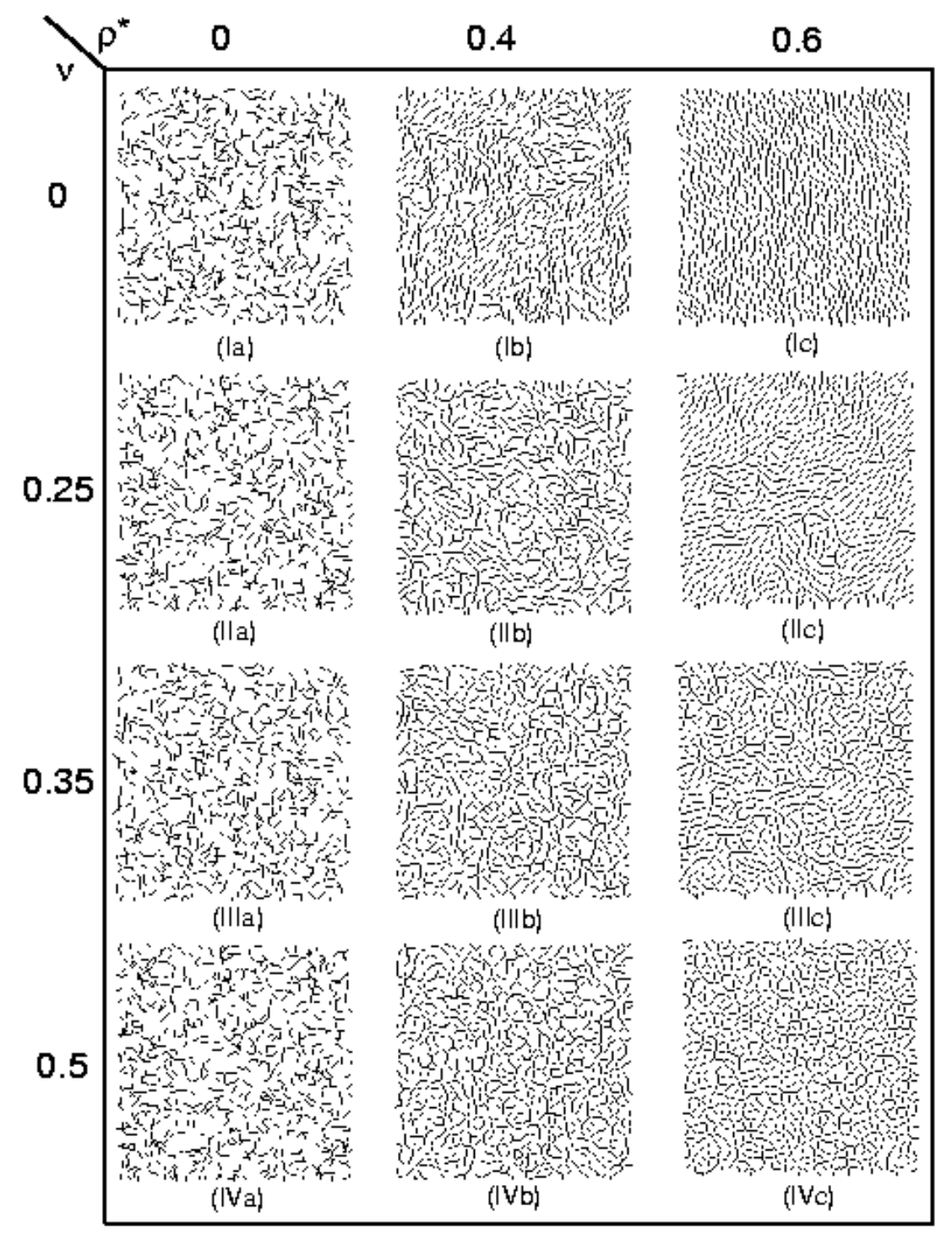

Figure 5: Bischofs and Schwarz 

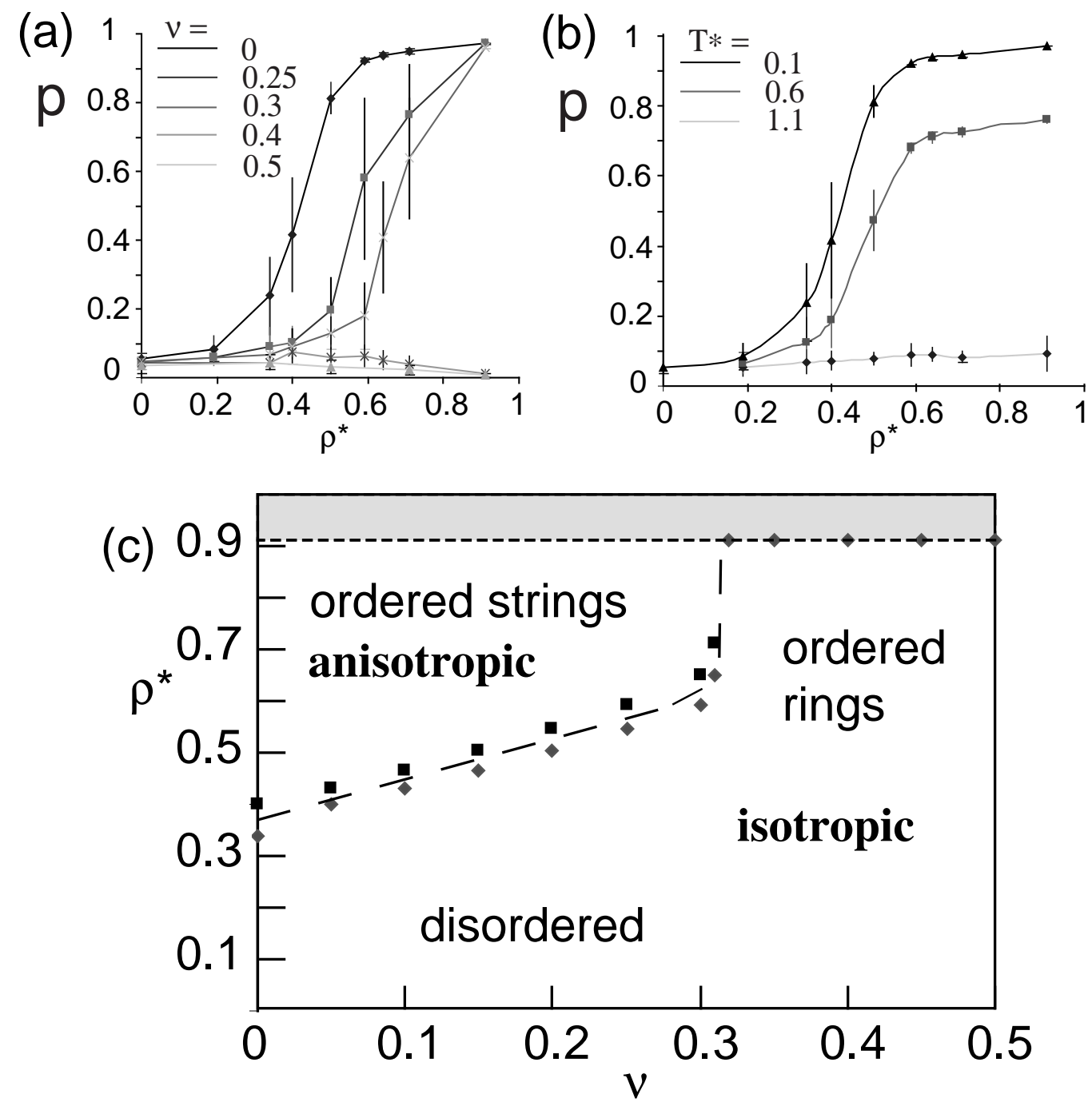

Figure 6: Bischofs and Schwarz 

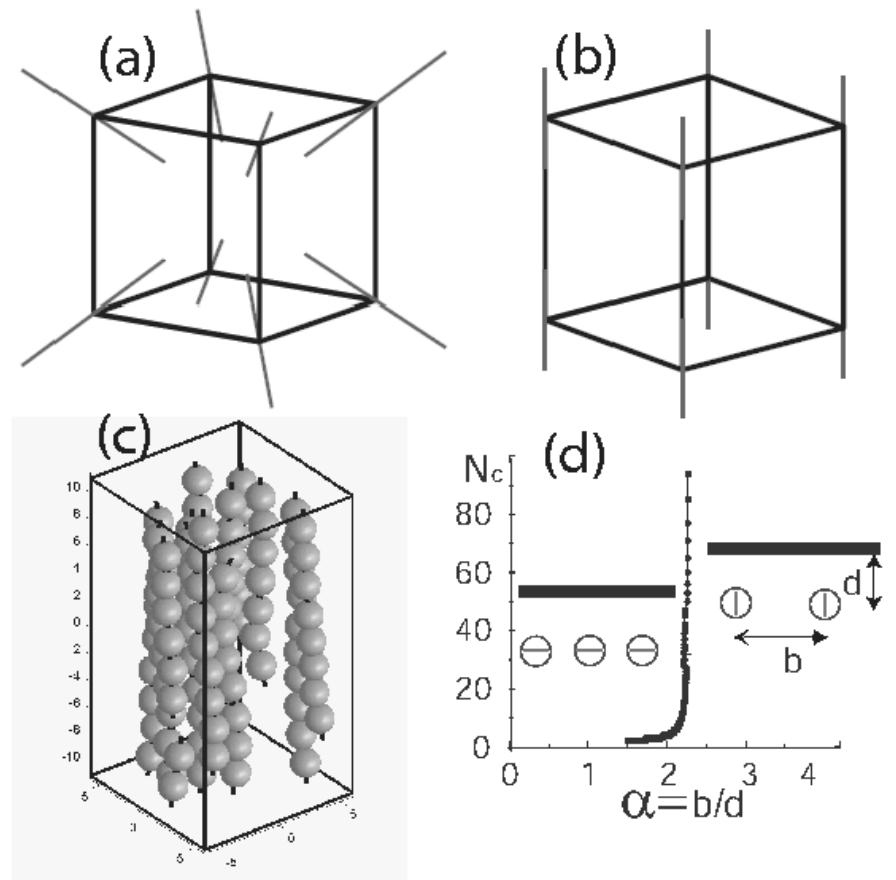

Figure 7: Bischofs and Schwarz 